\title{
EXISTENCE AND MULTIPLICITY OF SOLUTIONS FOR THE NONLINEAR KLEIN-GORDON EQUATION COUPLED WITH BORN-INFELD THEORY ON BOUNDED DOMAIN
}

\author{
KAIMIN TENG
}

Abstract. In this paper, we prove some existence and multiple results for the following nonlinear Klein-Gordon equation coupled with Born-Infeld theory

$$
\begin{cases}\Delta u=\left(m^{2}-(\omega+\phi)^{2}\right) u-f(x, u), & \text { in } \Omega, \\ \Delta \phi+\beta \Delta_{4} \phi=4 \pi(\omega+\phi) u^{2}, & \text { in } \Omega, \\ u=\phi=0, & \text { on } \partial \Omega,\end{cases}
$$

where $\Omega \subset \mathbb{R}^{N}(N \geqslant 3$ ) is a bounded domain with smooth boundary, and $f \in C(\bar{\Omega}, \mathbb{R})$ satisfies some assumptions.

Mathematics subject classification (2010): 26D15, 26A51, 32F99, 41A17.

Keywords and phrases: Klein-Gordon equation, Born-Infeld theory, variational methods.

\section{REFERENCES}

[1] A. Azzollini, L. Pis Ani, A. Pomponio, Improved estimates and a limit case for the electrostatic Klein-Gordon-Maxwell system, to appear on Proc. Roy. Soc. Edinburgh Sect. A.

[2] A. Azzollini, A. Pomponio, Ground state solutions for the nonlinear Klein-Gordon-Maxwell equations, Topol. Methods Nonlinear Anal., 35 (2010), 33-42.

[3] V. Benci, D. Fortunato, Solitary waves of the nonlinear Klein-Gordon equation coupled with the Maxwell equations, Rev. Math. Phys., 4 (2002), 409-420.

[4] V. Benci, P. D'Avenia, D. Fortunato, L. Pisani, Solitons in several space dimensions: Derrick's problem and infinitely many solutions, Arch. Rational Mech. Anal., 154 (2000), 297-324.

[5] M. Born, L. Infeld, Foundations of the new field theory, Proc. R. Soc. Lond. A, 144 (1934), 425 451.

[6] T. D'Aprile, D. Mugnai, Solitary waves for nonlinear Klein-Gordon-Maxwell and SchrödingerMaxwell equations, Proc. R. Soc. Edinb. Sect. A, 134 (2004), 1-14.

[7] T. D'Aprile, D. Mugnai, Non-Existence results for the coupled Klein-Gordon-Maxwell equations, Adv. Nonlinear Stud., 4 (2004), 307-322.

[8] P. D' AVEnia, L. PIS ANI, Nonlinear Klein-Gordon equations coupled with Born-Infeld type equations, Electron. J. Diff. Eqns, 26 (2002), 1-13.

[9] D. Fortunato, L. ORsina, L. PisAni, Born-Infeld type equations for electrostatic fields, J. Math. Phys., 43 (2002), 5698-5706.

[10] D. MugnaI, Coupled Klein-Gordon and Born-Infeld type equations: looking for solitary waves, Proc. R. Soc. Lond. Ser. A Math. Phys. Eng. Sci, 460 (2004), 1519-1528.

[11] J. Polchins KI, TASI lectures on D-branes, arXiv:hep-th/9611050; R. ARgURIO, Brane physics in M-theory, hep-th/9807171; K.G. S AVVIDY, Born-Infeld action in string theory, hep-th/9906075.

[12] P. H. Rabinowitz, Minimax Methods in Critical Point Theory with Applications to Differential Equations, American Mathematical Society, CBMS Regional Conference Series in Mathematics, 1986. 
[13] W. A. Strauss, Existence of solitary waves in higher dimensions, Comm. Math. Phys., 55 (1977), $149-162$.

[14] M. Struwe, Variational Methods: Applications to Nonlinear Partial Differential Equations and Hamiltonian Systems, 3rd edition, Springer-Verlag, 2008.

[15] K. M. TENG, K. J. ZHANG, Existence of solitary wave solutions for the nonlinear Klein-Gordon equation coupled with Born-Infeld theory with critical Sobolev exponent, Nonlinear Analysis, 74 (2011), 4241-4251.

[16] M. Willem, Minimax Theorems, Progress in Nonlinear Differential Equations and their Applications, 24, Birkhäuser, Boston, 1996.

[17] Y. YU, Solitary waves for nonlinear Klein-Gordon equations coupled with Born-Infeld theory, Ann. I. Poincaré, 27 (2010), 351-376. 